\title{
CranioSacral Therapy and Visceral Manipulation: A New Treatment Intervention for Concussion Recovery
}

\author{
Gail Wetzler, PT, DPT, EDO, BI-D, ${ }^{1,2}$ Melinda Roland, MA, PT, LAc, OMD, Dipl-Ac, CST-D, ${ }^{3,4}$ \\ Sally Fryer-Dietz, PT, DPT, CDT-D, SIPT Cert, ${ }^{3,5}$ and Dee Dettmann-Ahern, PT, BI-D ${ }^{1,6}$
}

\begin{abstract}
Background: Military service members and veterans face health issues related to traumatic brain injury (TBI), especially during combat, use of heavy equipment, and exposures to environmental hazards and explosives. There were 400,000 TBIs reported in deployed U.S. troops in 2012. Athletes are also subject to TBI. Studies have indicated that some manual therapies could be helpful for treating patients who have post-concussive syndrome. Objective: This case series report describes the effects of CranioSacral Therapy (CST), Visceral Manipulation (VM), and Neural Manipulation (NM) modalities for treating patients who have post-concussion syndrome. The goal of this study was to evaluate these effects on immobility, pain intensity, quality of life, sleep disorders, and cognition in these patients.

Materials and Methods: This single-blinded case series was conducted at the Upledger Institute, in West Palm Beach, FL. The patients were 11 male retired professional football players from the National Football League and the Canadian Football League who had been medically diagnosed with post-concussion syndrome. Each participant received a morning and afternoon 2-hour session of these three specific manual therapies, which were capable of accessing and addressing the structural, vascular, and neurologic tissues of the cranium and brain - as well addressing far-reaching ramifications throughout the body following trauma. The main outcome measures were scores on the: Impact Neurocognitive Test; Dynavision ${ }^{\mathrm{TM}}$ Test; Short Form-36 Quality of Life Survey, Headache Impact Test, Dizziness Handicap Inventory; a numeric pain rating scale; orthopedic range of motion tests (ROM); and vestibular testing. Hours of sleep were also checked. These outcome measures were registered at baseline, after treatment, and after a 3-month follow up.

Results: Statistically significant differences were seen with a decrease in overall pain rating scale scores $(P=0.0448)$, and cervicogenic pain levels decreased $(P=0.0486)$. There were statistically significant increases in Dynavision Average Reaction Time $(P=0.0332)$, Memory Test $(P=0.0156)$ scores, and cervical ROM scores $(P=0.0377)$. Hours of sleep averaged 2 hours on the first day of treatment and increased to 4.0 hours at the end of treatment and were continuing to increase, as noted at a 3-month evaluation.

Conclusions: Ten sessions of specific CST/VM/NM therapy resulted in statistically greater improvements in pain intensity, ROM, memory, cognition, and sleep in concussed patients.

\footnotetext{
${ }^{1}$ Clinical Research, Barral Institute, West Palm Beach, FL.

${ }^{2}$ Wetzler Integrative Physical Therapy, Denver, CO.

${ }^{3}$ Clinical Research, Upledger Institute, West Palm Beach, FL.

${ }^{4}$ Integral Therapies, San Diego, CA.

${ }^{5}$ Integrative Concussion Therapy, Dallas, TX.

${ }^{6}$ Dee Ahern Physical Therapy, Stuart, FL.
}

(C) Gail Wetzler et al., 2017; Published by Mary Ann Liebert, Inc. This is an Open Access article distributed under the terms of the Creative Commons Attribution License, which permits unrestricted use, distribution, and reproduction in any medium, provided the original work is properly cited.
\end{abstract}


Keywords: concussion, traumatic brain injury, CranioSacral Therapy, Visceral Manipulation, Neural Manipulation, treatment intervention

\section{INTRODUCTION}

$\mathbf{M}$ ANY VICTIMS OF CONCUSSION INJURIES report signs and symptoms years after the incident(s). In this study, conducted in 2014 and 2015, a team of physical therapists, osteopaths, and allied health professionals discovered that specific manual therapy techniques applied to patients with post-concussive syndrome provided significant results for future concussion recovery. The patients in this study were retired professional football players from both the National Football League (NFL) and the Canadian Football League who were medically diagnosed with postconcussion syndrome. These initial outcomes were inspiring and showed real promise for viable treatment options for traumatic brain injury (TBI), concussion, and the secondary compensatory dysfunctions that occur in the days following TBI.

Military service members and veterans face these health issues as well, especially during the possibility of combat, use of heavy equipment, and exposures to environmental hazards and explosives. There were 400,000 TBI reported in deployed U.S. troops in 2012. ${ }^{1}$ Snell and Halter in a 2010 study stated that TBI, whether by direct impact or due to blast-wave effects, became the "signature wound" of the conflicts in Afghanistan and Iraq. ${ }^{2}$ While there are some overlapping elements, the pattern of injury that results from a blast-induced TBI is not the same as a sports-related concussion. In a recent study funded by the United States Army and from the Military Operational Medicine Research Program, the research on open-field blast exposure showed significant neuronal injury in the gray and white matter areas of the brain closest to the blast, unique skull fractures, diffuse cerebral edema, and compressive forces to the heart that eventually causes a blood surge in the brain (Fig. 1). ${ }^{3}$

During this same time period, the severity of symptoms the retired football players were dealing with following concussion and the potential outcome of chronic traumatic encephalopathy was unbearable, and some men were committing suicide. ${ }^{4,5}$

In 2011, Amen et al. had published the first and largest brain imaging study using single-photon emission computed tomography and quantitative electroencephalography with 100 active and retired NFL players. That study reported consistent damage to the brain's prefrontal cortex (judgment, impulse control), temporal lobes (memory, mood stability), parietal (spatial orientation) occipital (visual field) areas, and the cerebellum (mental agility, coordination, speed). ${ }^{6}$ The consistency of finding these damaged areas in the brain plus the public outcry for help, created curiosity regarding studying the modalities of CranioSacral Therapy (CST), Visceral Manipulation (VM), and neural manipulation (NM), and their effects on patients who have TBI.

\section{MATERIALS AND METHODS}

The purpose of this research was to determine if specific manual therapy techniques would comprise an intervention that would induce positive changes to enhance recovery or diminish long-term symptoms for professional athletes with histories of concussions. This purpose was based on a recent study on the practice parameters of sports concussions, published in Neurology in 2013 by Giza et al. Giza's team asked: "What interventions enhance recovery, reduce the risk of recurrent concussion or diminish longterm sequelae?",7

The Upledger Institute (UI) in West Palm Beach, FL, and the Ricky Williams Foundation in Austin, TX, provided a platform to answer this question and begin a Concussion Pilot Program (CPP). The CPP team consisted of a medical director, a research director, a program coordinator, a psychotherapist, and a team of certified therapists qualified in these techniques. With the recognition that the chronicity of this condition has several determinants, multidisciplinary

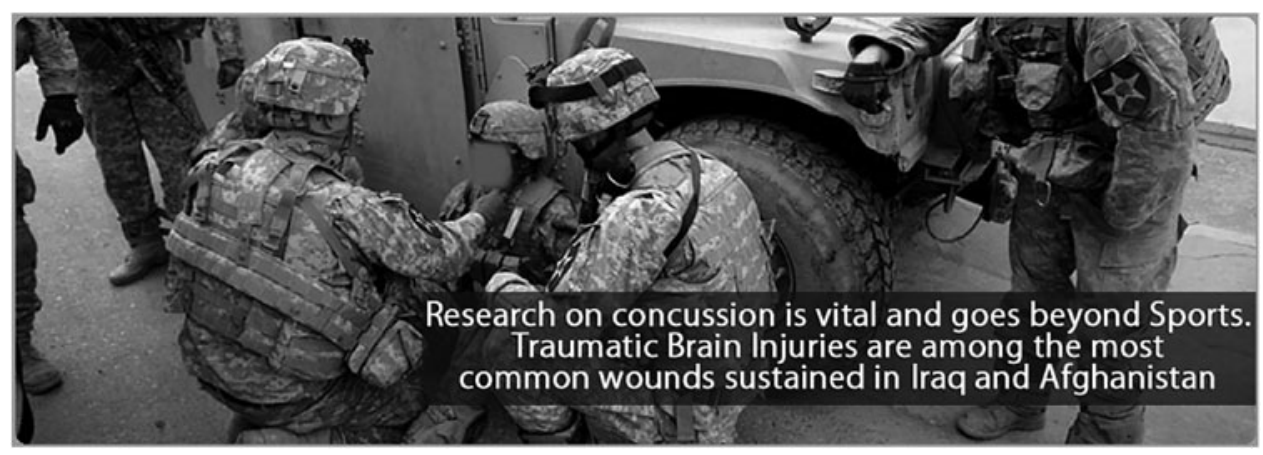

FIG. 1. Concussion injuries in the military in 2012. 


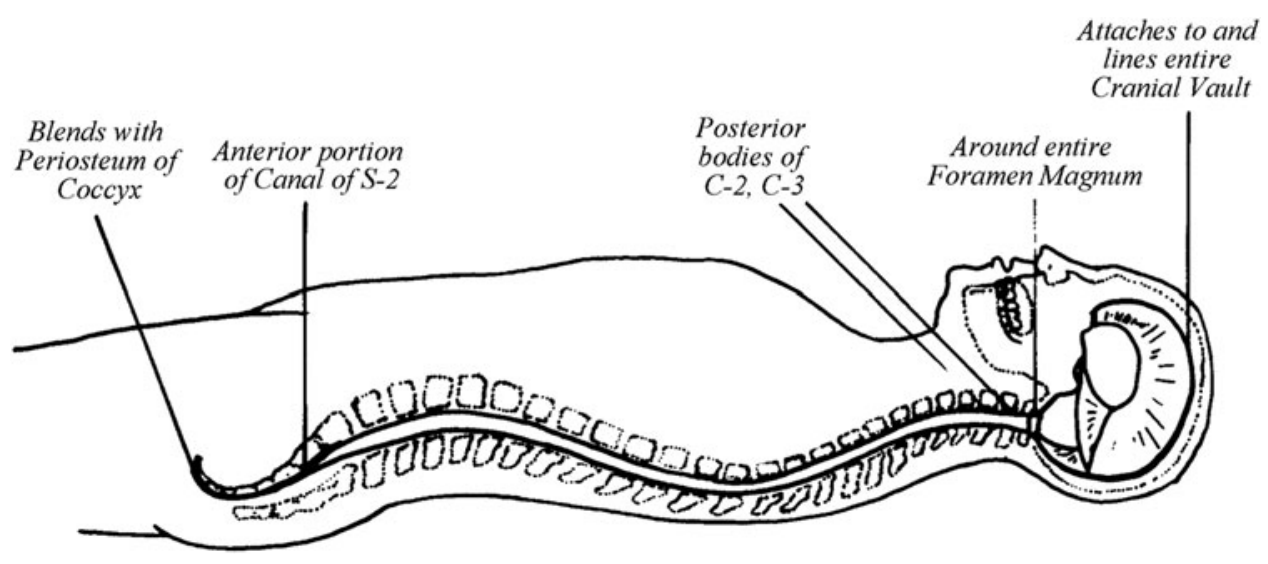

FIG. 2. Anatomy illustration of the craniosacral system.

management of patients suffering from post-concussion syndrome has now been widely adopted. ${ }^{8}$

The tests and treatments took place at the UI. The participants were recruited by Ricky Williams (a former NFL running back), and they all signed informed consent waivers to be in this study. The inclusion criteria consisted of retired professional football players with histories of previous head injuries. The exclusion criterion was an acute or recent head injury. The medical director of the UI reviewed the protocol for this study. A total of 11 applicants were accepted into it. Each participant received a medical and neurologic examination by the UI medical director confirming the diagnosis of post-concussive syndrome. No neuroimaging was administered during this pilot program; however 3 of the participants did have prior neuroimaging scans that revealed the presence of structural abnormalities.

The professionals involved with this study had many collective years of experience working with patients having TBI, autism, post-traumatic stress disorder, anxiety, depression, chronic headaches, chronic pain, memory loss, and sleep disorders. The treatment modalities used were developed in the mid-1970s; thus, a 40-year history of clinical expertise existed. These manual therapies are based on the osteopathic principles that structure and function are interrelated, the body is a unit (united by fascia-the biologic "fabric" that surrounds all cells of the body), and that the body is a self-correcting mechanism. Supporting the body's inherent self-corrective mechanism enables a return of optimal function and thus health and well-being.

Upledger CST is a gentle, noninvasive manual therapy that modifies and corrects restrictions in the craniosacral system (CSS). The CSS consists of meninges, bones attached to the meninges, cerebrospinal fluid (CSF), and CSS glia. The CSS surrounds, protects, nourishes, and cleanses the brain and spinal cord. The essential part of CST is to optimize the flow of CSF into and out of the central nervous system (CNS). ${ }^{9}$ Restrictions within the CSS can affect its components and the normal physiology of the brain and spinal cord adversely, such as motor and sensory signaling or autonomic nervous system (ANS) interactions. Research over the past 35 years has shown that glial cells are essential for neuronal signaling and CNS development, controlling CNS blood flow and CSF production/flow, and maintaining brain homeostasis (Fig 2). ${ }^{10,11}$

Box 1 shows five principal concepts that connect the three therapies used.

Barral VM is a manual therapy consisting of gentle-yet specifically placed-manual forces that encourage normal mobility, vascularity, tone, pressure, and inherent tissue motion of organs; their connective tissues; and their relationship to other areas of the body where physiologic motion has been impaired. The central premise of VM is that an organ in good health requires physiologic motion. Should an organ, including the brain, lose its mobility or its inherent tissue motion (motility), then barriers to the physiologic mechanisms of the body develop. VM is the primary modality used to correct these tensions and restore movement within the vascular, digestive, eliminatory, respiratory, reproductive, and lymphatic systems as well as the ANS. ${ }^{12}$

Barral NM focuses on releasing local nerve restrictions to help restore and balance the freedom of movement for

Box 1. Five Principal Concepts of CST, VM, and NM

Connecting relationships of the modalities chosen in the treatment approach for post-concussion syndrome include the following concepts:

(1) In order for tissues to survive and maintain equilibrium, the connection between each tissue must be free to move in its environment.

(2) The tissue must have substantial mobility, extensibility, and elasticity.

(3) When a tissue loses equilibrium, physiology is compromised.

(4) Symptoms can be local or distant from their origin.

(5) Symptoms are not exclusive to physical structures.

CST, CranioSacral Therapy; VM, visceral manipulation; NM, neural manipulation. 
intracranial pressures and the central or peripheral nerves. The manual therapist evaluates pre and post comparisons of dural membrane viscoelasticity, pre and post cranial bone articular movement tests, and pre and post cranial rhythm impulse tests in order to ascertain if movement has been restored. VM is also a lighter touch manual therapy that is used to examine how the release of these local nerve fixations resolves the more comprehensive (global) dysfunctional patterns. Mechanical properties of nerves require mobility, elasticity, and a compliant intrinsic and extrinsic intraneural pressure system. The nervous system is a functional unit that relies on electrical impulse, mechanical continuity via fascial tissue envelopes, biomechanical communication via centrifugal and centripetal axon-plasmic flux, and electromagnetic frequency as a carrier of continuous currents (initiated by brainwaves), which diffuse through the neural network to the interior of the body. ${ }^{13}$

According to the 4th International Consensus Statement on Concussion in Sport, the American Academy of Neurology (2013) and Centers for Disease Control and Prevention (2013), concussion is defined as an alteration of brain function caused by a direct or indirect impulsive force to the body or skull with or without loss of consciousness (LOC). ${ }^{14}$ According to Grady et al.'s work from 2012, changes in the ion channels occur from membrane deformation caused by trauma to the head. Widespread depolarization, causing neuron suppression, potassium efflux and calcium influx, causes impairment of adenosine triphosphate production in the mitochondria, requiring higher levels of glucose metabolism. This imbalance causes axonal swelling, increased membrane permeability, and cerebral edema. ${ }^{15}$ Calcium ions represent the intracellular message for brain integration and are the main drivers of glial cells for blood flow, and comprise the mechanism to facilitate the brain's resolution of edema. ${ }^{16}$

The above modalities are capable of being used to evaluate and treat the structural, vascular, and neurologic tissues associated with a concussion injury, as well as the farreaching ramifications throughout the body following such trauma. It is the knowledge of anatomy, the connection of fascial networks, the recognition of tissue restrictions, and the infinite wisdom of tissue intelligence that guides the manual mechanical dialogue inherent in these modalities. CST has been studied for treating fibromyalgia, ${ }^{17}$ temporomandibular disorders, ${ }^{18}$ migraine, ${ }^{19}$ lateral epicondylitis, ${ }^{20}$ dementia, ${ }^{21}$ multiple sclerosis, ${ }^{22}$ chronic low-back pain, ${ }^{23}$ and chronic neck pain. ${ }^{24}$

\section{Design}

This study was designed as a new treatment approach for a special population. In this intensive therapy program, each participant received treatment using specific CST/VM/NM techniques, twice per day, for 5 days. Each participant received an initial evaluation in the morning of day 1 , a post- evaluation in the afternoon of day 5, and a follow-up evaluation at 3 months. The participants were eleven retired professional male football players. The number of concussions for each participant ranged from 3 to $100+$, with LOC reported in 6 of the 11 retired players. There were 5 participants in the 2014 study and 6 participants in the 2015 study. Their ages ranged from the mid-20s to the mid-70s.

\section{Test Methods}

The standard manual therapy evaluation for cranial tissue restrictions must take into consideration four possible contraindications for CST (see Box 2). The four specific conditions for which CST is clinically contraindicated are (1) acute intracranial hemorrhage, (2) intracranial aneurysm, (3) a recent skull fracture, and (4) any herniation of the medulla oblongata. None of the 11 participants had these complications and the MD who examined the participants for acceptance into this study cleared all 11 .

The chief complaints among the participants with postconcussion syndrome had consistent similarities (Table 1):

- Head/cervical pain (80\%)

- Depression $(80 \%)$

- Difficulty with memory, learning, reading, and decision making $(80 \%)$

- Sleep problems $(60 \%)$

- Fatigue (60\%)

- Anxiety (40\%)

- Dizziness/balance problems (20\%)

- Digestive/elimination problems (10\%).

As the therapists became acquainted with the individual patterns of dysfunction, the findings were shared with each

\begin{tabular}{l} 
Box 2. TESTS AND MEASUREMENTS \\
\hline The tests and measurements used consisted of a \\
combination of "gold standard" concussion tests found in \\
the literature and standard manual therapy tests for \\
connective tissue restrictions. The tests included: \\
(1) Medical examination \\
(2) Impact/Neurocognitive Test/Dynavision ${ }^{\mathrm{TM}}$ Test \\
(3) Short Form-36-Quality of Life Survey \\
(4) Headache Intensity Test \\
(5) Dizziness Handicap Inventory \\
(6) Range of motion tests for involved joints and cervical \\
(7) Vestibular and balance tests \\
(8) Numeric pain rating scale (number given for overall \\
(9) Quantity/hours of sleep \\
(10) Cranial Rhythm Impulse Test \\
(11) Cranial Membrane Tension Test \\
(12) Peripheral and Cranial Nerve Mobility Test \\
(13) Visceral Mobility and Motility Test \\
(14) Fascial Listening Test to find primary driver of \\
\end{tabular}







participant and goals were discussed. Studies in neuroscience have shown that, when working with trauma, it is an important step in the healing process when a person has a better understanding of his or her personal situation (selfawareness/mindfulness). ${ }^{25}$ Therefore, the participants gathered as a larger group to learn about the therapies each was about to receive and how these modalities have been found to calm the threat felt by the nervous system and assist the coping mechanisms for handling trauma. The therapists explained that the goal of these modalities was to break the pattern of excessive nerve firing, vascular insufficiency, autonomic responses, anxiety related to these stressors, sleeplessness, inflammation, and muscle tensions. All of these issues were leading to the chronic pain, fatigue, dizziness, headaches, depression, memory loss, and quality of life (QoL) issues that were the chief complaints of these participants.

The intervention consisted of these manual therapy techniques given by certified, licensed and experienced

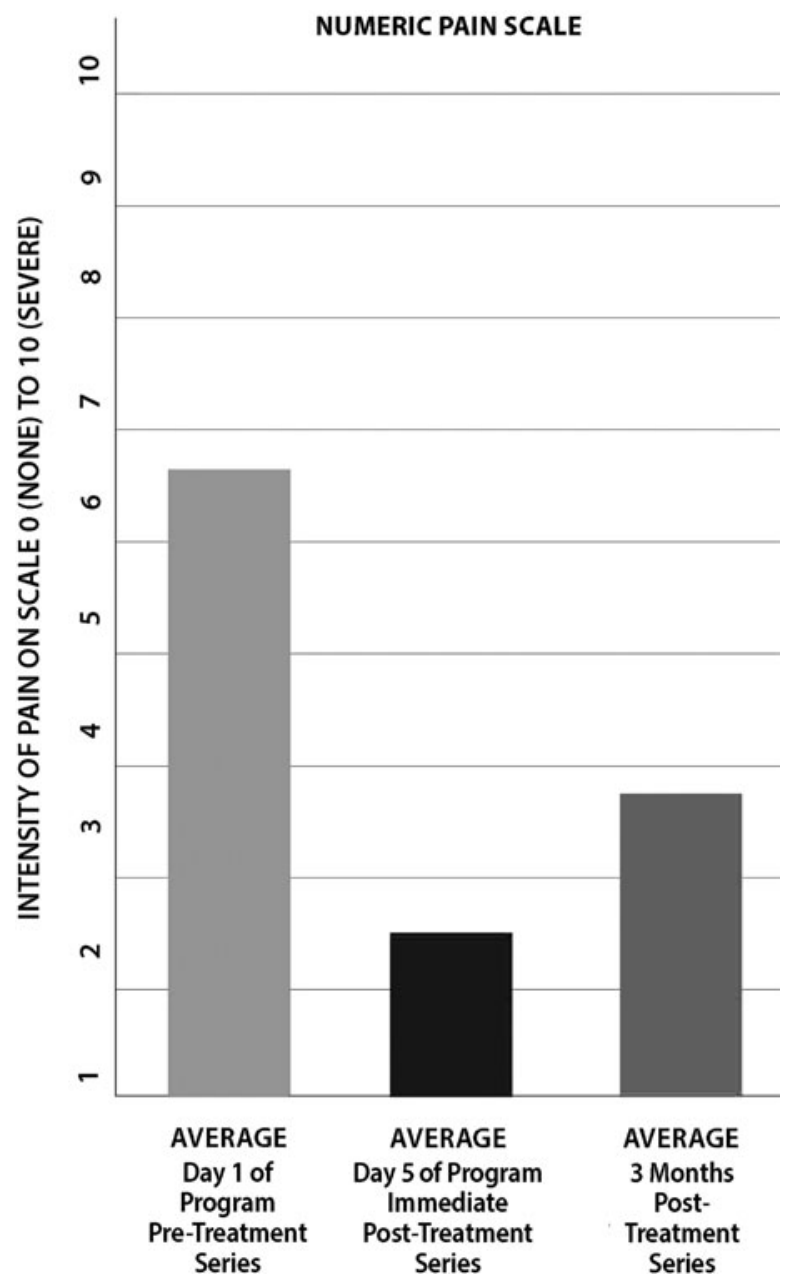

FIG. 3. Numeric pain scale. This graph illustrates a subjective self-evaluation of a subject's overall perceived pain level. A decrease in this participant's pain was statistically significant $(P=0.0448)$. therapists at the UI. Each session was individually based on the participant's baseline test results, therefore therapists could choose to administer just one or two of the three possible treatments. There also was the potential for more than 1 therapist to treat a patient in the same session. The therapy sessions took an average of 90-120 minutes each. The program began each morning with a group discussion with all participants and therapists. The program ended each evening with a meeting among the therapists to discuss progress and treatment strategies.

\section{RESULTS}

Although there was standard measurement in this study, it was patient-centered, given that no two concussions are the same, even for a single athlete. ${ }^{26}$

The graphs in Figures 3-9 are illustrated outcomes of this study. The comparisons showed a statistically significant

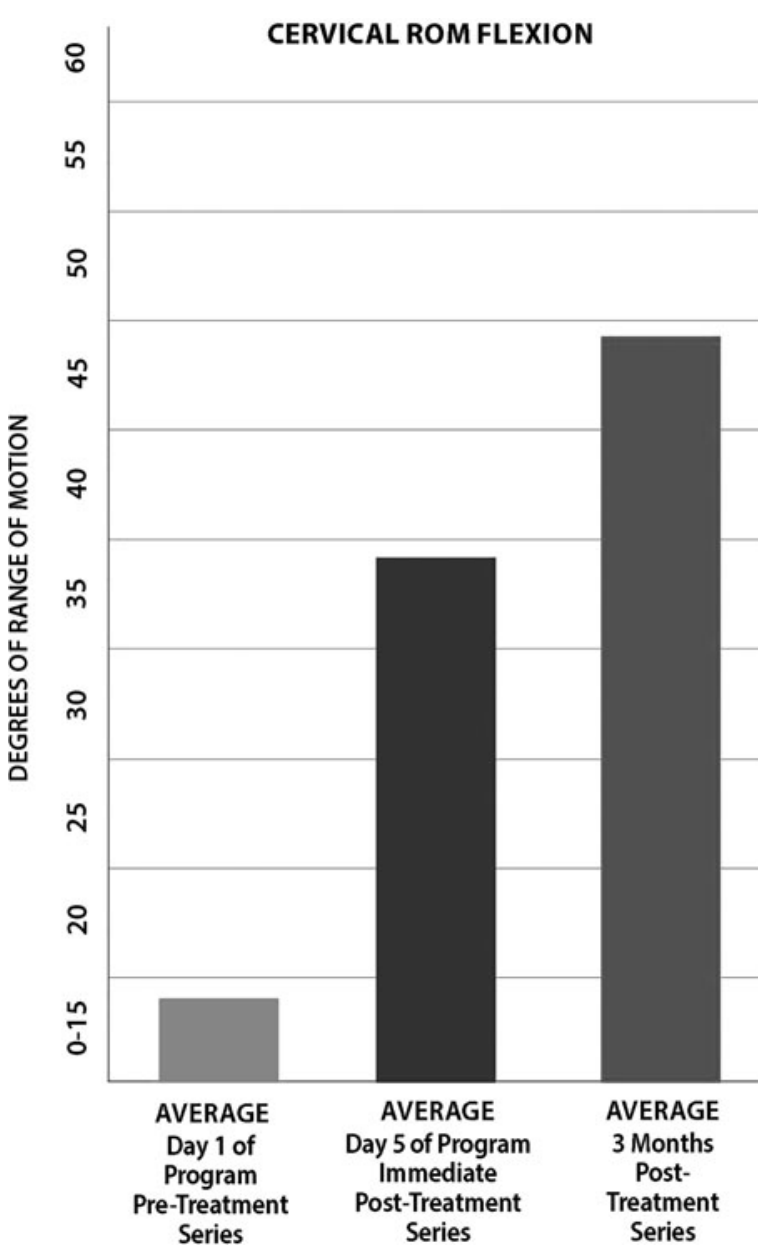

FIG. 4. Cervical range of motion (ROM). This graph illustrates the average degree of ROM in a participant's cervical spine. Here, $0^{\circ}-15^{\circ}$ would be a low degree of movement while $40^{\circ}-60^{\circ}$ would be a high degree of motion. Statistically significant changes $(P=0.0377)$ occurred. 


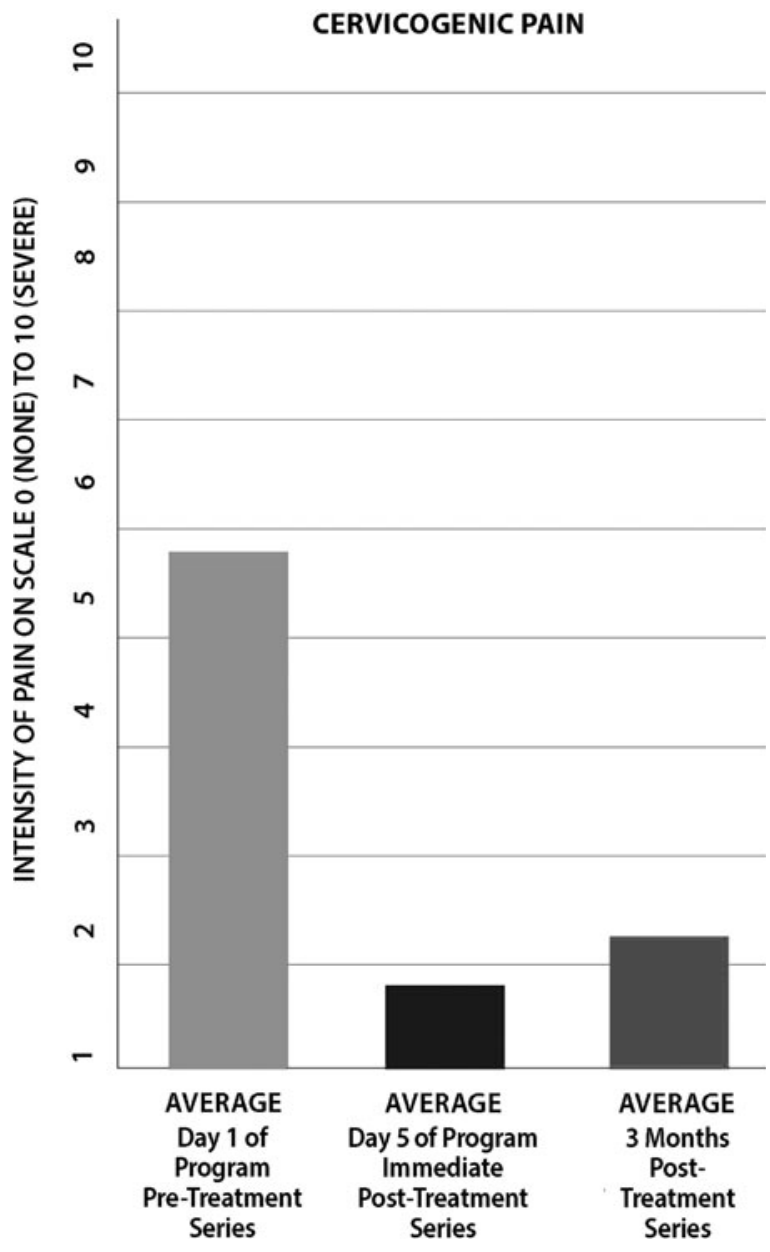

FIG. 5. Cervicogenic pain $(P=0.0486)$. Manual therapy techniques for the treatment of chronic neck pain show a statistically significant decrease in this participant's pain levels.

decrease in the participants' overall pain scores $(P=$ $0.0448)$. The active ROM score was statistically significant $(P=0.0377)$ helping the participants' cervicogenic pain levels decrease $(P=0.0486)$. The memory scores $(P=$ $0.0156)$ as well as the neurocognitive average reaction time scores $(P=0.0332)$ were also statistically significant. At follow-up, the number of hours slept per night as well as QoL issues (general health, bodily pain, energy, emotional well-being, and social functioning) significantly increased.

The independent manual therapy testing of connective tissue restrictions showed that specific manual therapy techniques would comprise an intervention with positive changes to enhance recovery of long-term sequelae for postprofessional athletes with histories of concussions (Table 2).

\section{DISCUSSION}

The results of this preliminary study suggest that ten consecutive sessions of CST, VM, and NM resulted in a

\section{DYNAVISION TEST RESULTS}

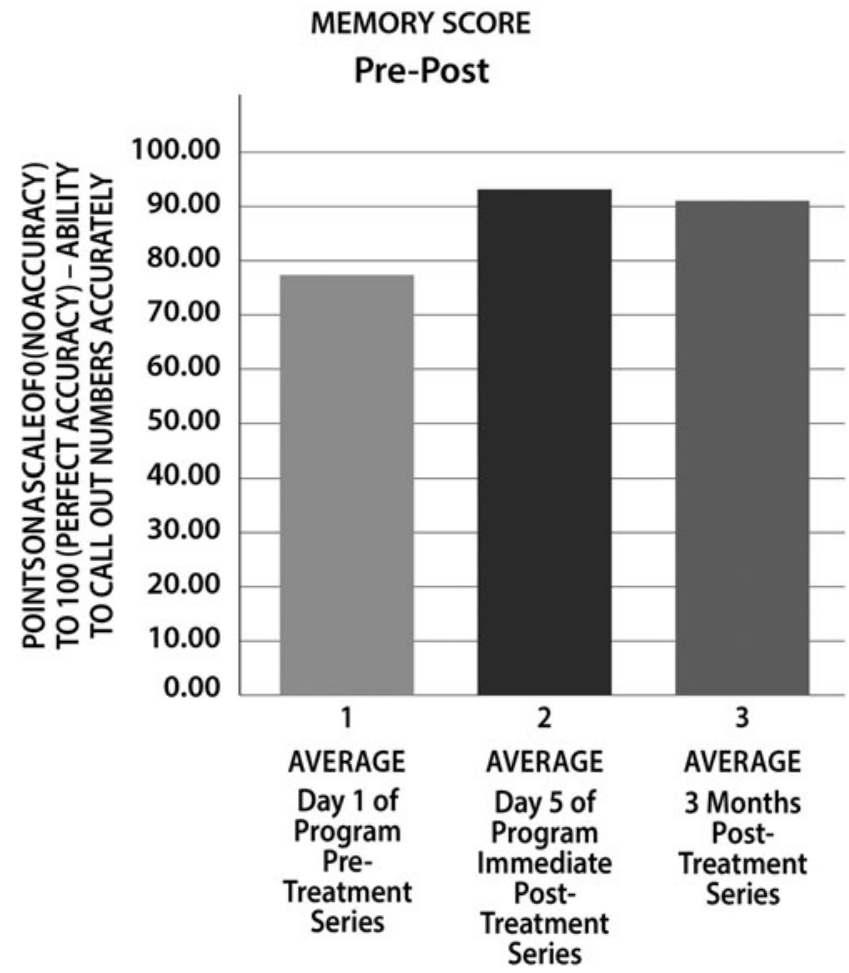

FIG. 6. Memory score $(P=0.0156)$. The Dynavision $\mathrm{D} 2^{\mathrm{TM}}$ testing allows for testing sequential memory, focus, and concentration. The test utilizes three memory tests of graded complexity to increase cognitive demands quickly. Statistical analysis shows memory test scores improved significantly from pre-test to posttests in a group average and continued to maintain this improvement throughout the 3 months that followed.

statistically significant reduction in overall pain that was particularly significant for pain arising from the neck region. Given that this group reported $80 \%$ of pain experienced was centered in the neck and head region, this statistical change represented freedom from higher pain levels (Cervicogenic Pain at 6/10 to 2/10) and helped increase the participants' cervical ROM (from $18^{\circ}$ to $48^{\circ}$ within the week). Their sleep time doubled, which helped reduce the fatigue and anxiety reported in $60 \%$ of this patient population. The significant reaction time changes were seen throughout the week in therapy (balance or memory activities). With a statistically significant increase in the average memory score, less difficulty with learning, reading, and making appropriate decisions was manifested in the gains the men made with the QoL issues, especially depression (as noted on the SF-36 scale).

When the average reaction time did show a significant decrease in time needed to do a task, the visual reaction time did not make a statistically significant difference. The dynamic gait index test and the Timed Up and Go (TUG) test-standard in neurologic evaluations-did not prove to be significant in this patient population. The visual reaction 


\section{DYNAVISION TEST RESULTS}
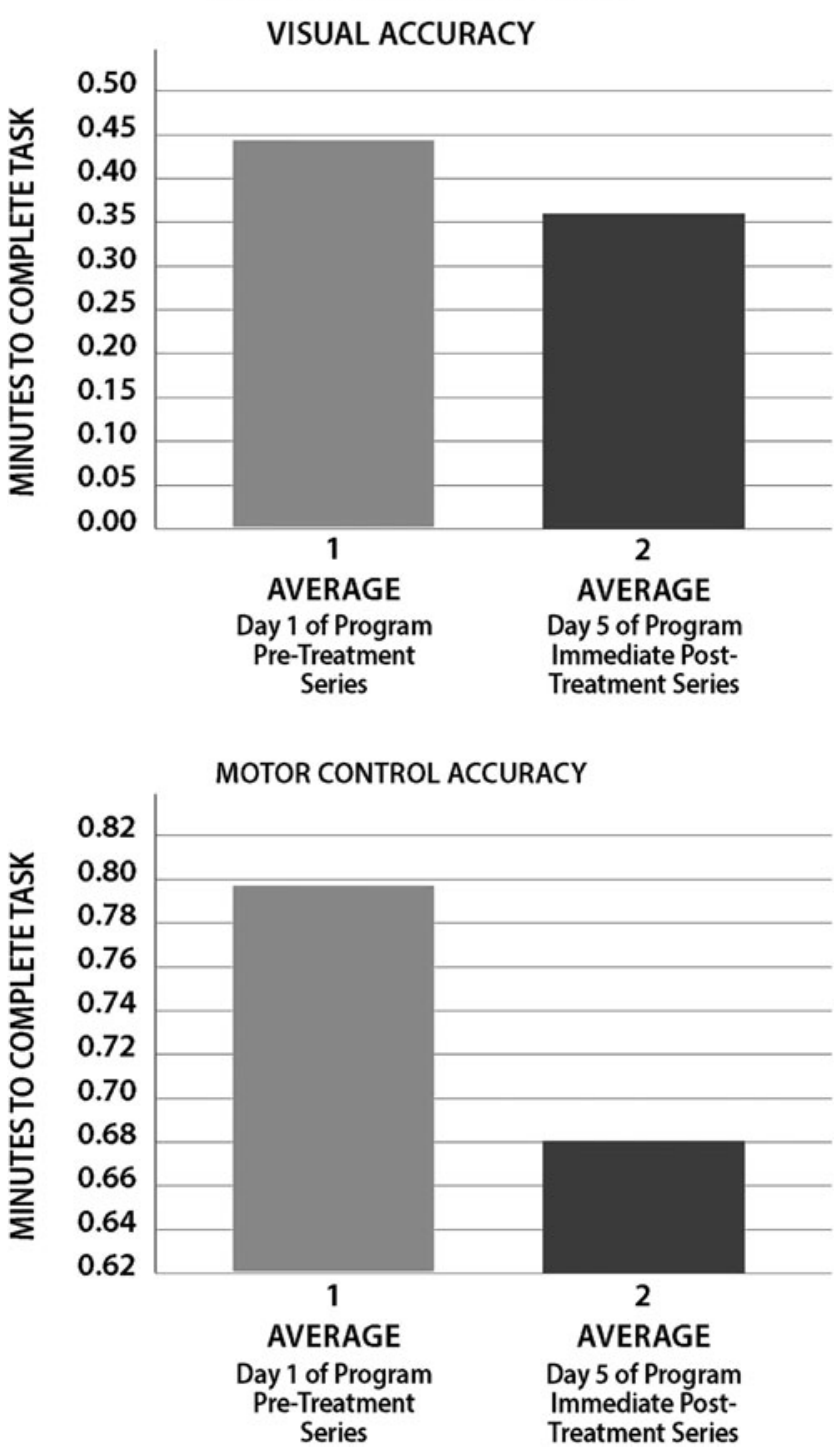

FIG. 7. Visual and average reaction time $(P=0.0332)$. The average visual and reaction time reflects the completion of perceptual processing. While the average visual reaction time was not significant the average reaction time (motor control) showed a statistically decrease in average reaction time.

time testing results showed a need to consider different testing techniques to assess each participant's vestibular system, with correlated dysfunctions and recovery. Reevaluating the efficacy of using both the dynamic gait test and TUG test was necessary, based on this study population. The participants did not show delays, even in initial evaluations, because of previous athletic conditioning.

The therapists concluded that post-concussion syndrome does not seem to be isolated to the brain. The independent fascial tests showed the dural membrane restriction affecting the lumbar/sacral region and thoracic spine with its organ contents to have secondary compensatory dysfunctions that occur in this syndrome. Each participant initially

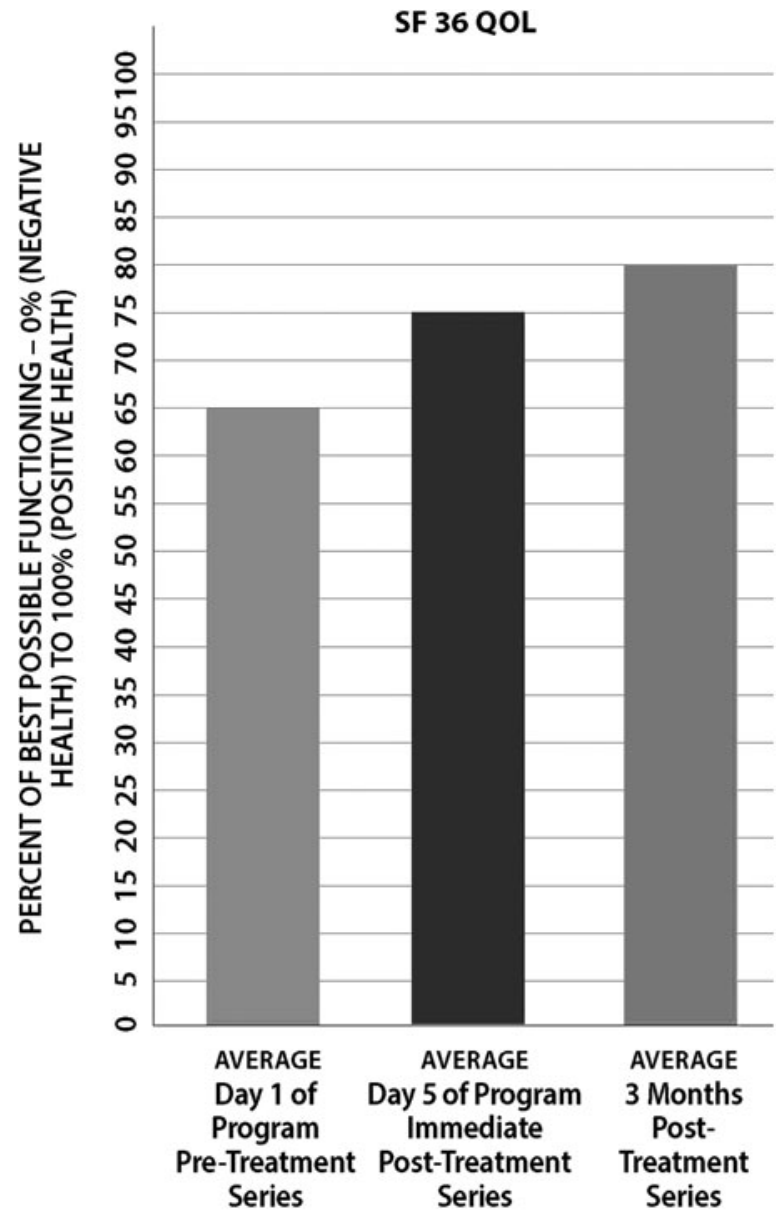

FIG. 8. Short Form-36 (SF-36) Quality of Life (QoL) Survey. The SF-36 is a generic, self-administered survey of a patient's perceived health/QoL. The survey measures physical function, vitality, social function, limitations due to emotional problems, general mental health, and health transition.

reported varied and random pain throughout his body, and every participant reported that previous joint or muscle pain had become elevated after the concussion. These symptoms were minimized post-treatment.

The current study had several limitations. First, the number of participants was small in this study as the Upledger Foundation is not a large facility and funding was limited. A larger number of participants would provide stronger statistical significance to support the data. Second, as a result of this small patient base, there was an inability to have controls. Third, the intervention with CST can be given by 1 practitioner or multiple practitioners in the same session, which might have limited the generalizability of the results.

The findings in this study agree with Ries, a boardcertified specialist in orthopedic physical therapy and brain injury, who said: "Concussion injury is a recoverable injury," especially when it "is viewed from an enablement rather than a disablement model." 8 Post-concussion syndrome is challenging but, with a multidimensional team approach, the research therapists are encouraged regarding 


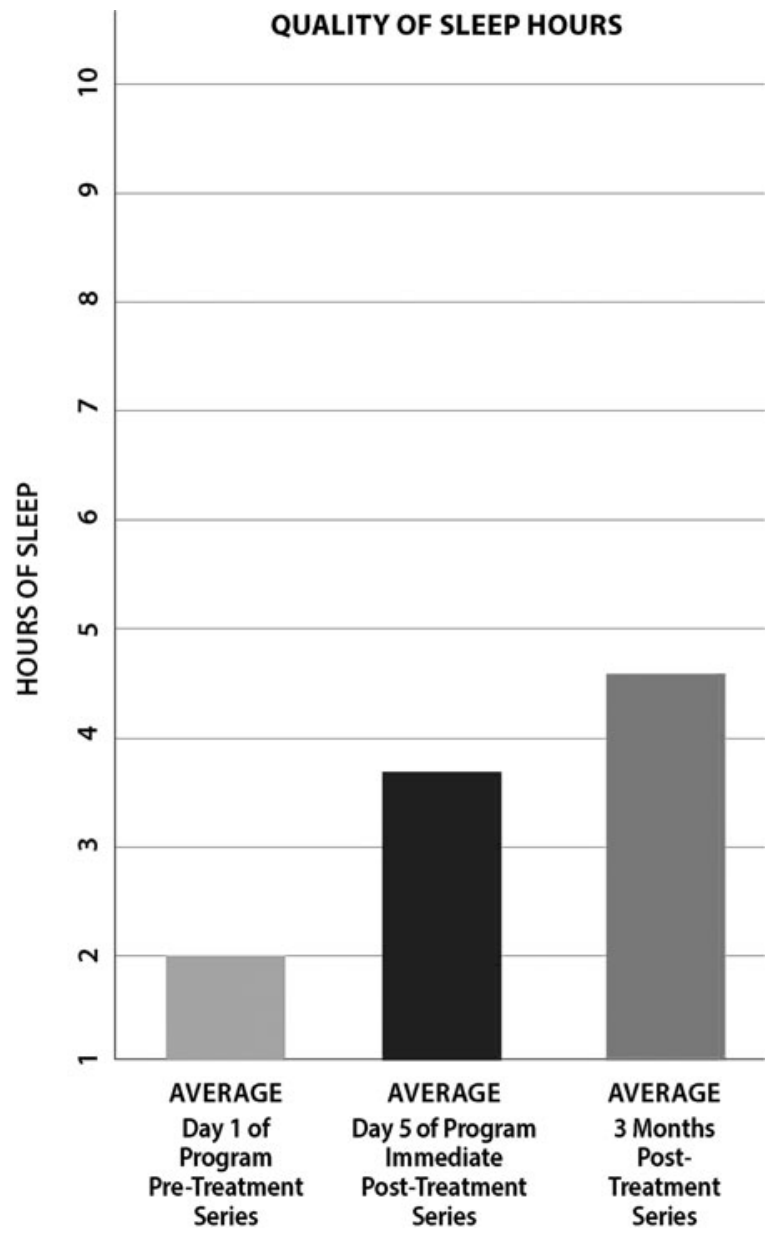

FIG. 9. Average hours of sleep per night. As fatigue and sleep deprivation consisted of $60 \%$ of the participants' chief complaints, it was possible to see an appreciable gain in sleep hours during 5 days of treatment, a gain that continued to increase in the months that followed.

the possibility of viable treatment options that can be taken into other clinics for concussion rehabilitation. The group discussions at the end of each day added a new dimension to the men's recovery. Many participants thought they were isolated with their feelings, symptoms, and problems. During the discussions, a new attitude of sharing, caring, and giving was identified. It was like being back on the team. One can practice skills and imagine the movements/drills, but until one starts playing the game-that is when it all comes together. Game on!

The current authors are encouraged by the results that specific manual therapies could induce improved health and healing from acquired brain injuries. This experience helped the therapists become aware that concussive injuries relate to whole-body trauma patterns. The ease with which each participant could move, the softening of volatility, and the often-dramatic decrease in pain is testimony to the gentle, yet profound, effect that the concussion treatment utilized in this study had on the restoration of balance in each man's body.
Table 2. General Findings for Connective Tissue Restrictions Within Study Participants

\begin{tabular}{lcc}
\hline Findings & Pre (Day 1) & Post (Day 5) \\
\hline $\begin{array}{l}\text { Participants with triad of } \\
\text { compression (lumbosacral } \\
\text { junction, occipito-atlantal } \\
\text { junction, \& sphenoid base) }\end{array}$ & $11 / 11$ & $3 / 11$ \\
$\begin{array}{l}\text { Fascial restrictions within the } \\
\text { thoracic cavity }\end{array}$ & $11 / 11$ & $4 / 11$ \\
$\begin{array}{l}\text { Loss in symmetry, quality, } \\
\text { amplitude \& rate of CRI }\end{array}$ & $10 / 11$ & $3 / 11$ \\
$\begin{array}{l}\text { Significant loss of cranial, } \\
\text { osseous \& suture flexibility }\end{array}$ & $9 / 11$ & $2 / 11$ \\
$\begin{array}{l}\text { Dural membrane restriction at } \\
\text { the foramen magnum } \\
\text { cranial nerves with fascial } \\
\text { restrictions-trigeminal, optic } \\
\quad \& \text { vagal }\end{array}$ & $9 / 11$ & $1 / 11$ \\
$\begin{array}{l}\text { organs with loss of mobility } \\
\text { and motility-heart, lungs \& } \\
\text { kidneys }\end{array}$ & $7 / 11$ & $4 / 11$ \\
\hline
\end{tabular}

CRI, cranial rhythm impulse.

\section{CONCLUSIONS}

Ten sessions of specific CST/VM/NM resulted in a statistically greater reduction in pain intensity, and greater improvements in mechanical ROM, memory, physical reaction time, and duration of sleep in patients with postconcussion up to 3 months after the intervention. This study was a preliminary study to answer the question asked by Giza et al.: "Which interventions enhance concussion recovery"? ${ }^{7}$ Future trials should investigate further the effectiveness of these specific manual therapies in patients with TBIs/concussion, especially military service members and veterans in addition to the population in this initial study.

\section{ACKNOWLEDGMENTS}

This research was supported by the John E. Upledger Foundation and the Ricky Williams Foundation.

\section{AUTHOR DISCLOSURE STATEMENT}

No financial conflicts exist.

\section{REFERENCES}

1. MedlinePlus. Traumatic Brain Injury. Online document at: www.medlineplus.gov/traumaticbraininjury.html Accessed February 6, 2017. 
2. Snell FI, Halter MJ. A signature wound of war: Mild traumatic brain injury. J Psychosoc Nurs Ment Health Serv. 2010;48(2):22-28.

3. Kallakuri S, Desai A, Feng K, et al. Neuronal injury and glial changes are hallmarks of open field blast exposure in swine frontal lobe. PLoS One. 2017;12(1):e0169239.

4. Iverson GL. Chronic traumatic encephalopathy and risk of suicide in former athletes. Br J Sports Med. 2014;48(2):162165 .

5. Webner D, Iverson GL. Suicide in professional American football players in the past 95 years. Brain Inj. 2016;30(1314):1718-1721.

6. Amen D, Newberg A, Thatcher R, Jim Y, Wu J, Keator $\mathrm{D}$, Willeumier K. Impact of playing American professional football on long-term brain function. $J$ Neuropsychiatry Clin Neurosci. 2011;23(1):98-106.

7. Giza C, Kutcher J, Ashwal S, et al. Summary of evidencebased guideline update: Evaluation and management of concussion in sports. Report of the Guideline Development Subcommittee of the American Academy of Neurology. Neurology. 2013;80(24):2250-2257.

8. Ries E. Beyond Rest: Physical Therapists and Concussion Management. PT in Motion, March 2017:22-27. Online document at: www.apta.org/PTinMotion/2017/3/Feature/BeyondRest/ Accessed June 5, 2017.

9. Upledger JE, Vredevoogd J. Introduction to the CranioSacral concept. In: CranioSacral Therapy. Seattle, WA: Eastland Press; 1983:5-13.

10. Wanveer T. Introduction and a few key words. In: Brain Stars, 1st ed. West Palm Beach, FL: Upledger Productions; 2015:1-30.

11. Nedergaard M, Rodriguiz J, Verkhratsky A. Glial calcium and diseases of the nervous system. Cell Calcium. 2010;47(2): 140-149.

12. Barral JP, Mercier P. Basic Concepts. In: Visceral Manipulation. Seattle: Eastland Press; 1988:1-20.

13. Barral JP, Croibier A. Some preliminary thoughts. In: Manual Therapy for the Cranial Nerves, 1st ed. London: Churchill Livingstone; 2008:1-5.

14. McCrory P, Meeuwisse WH, Aubry M, et al. Consensus statement on concussion in sport: The 4th International Conference on Concussion in Sport held in Zurich, November 2012. Br J Sports Med. 2013;47(5):250-258.

15. Grady MF, Master CL, Gioia GA. Concussion pathophysiology: Rationale for physical and cognitive rest. Pediatr Ann. 2012;41(9):377-382.

16. Ren Z, Iliff J, Yang L, et al. "Hit \& Run" model of closedskull traumatic brain injury (TBI) reveals complex pattern of post-traumatic AQP4 dysregulation. J Cereb Blood Flow Metab. 2013;33(6):834-845.

17. Castro-Sanchez AM, Mataran-Penarrocha GA, SanchezLabraca $\mathrm{N}$, et al. A randomized controlled trial investigating the effects of CranioSacral therapy on pain and heart rate variability in fibromyalgia patients. Clin Rehabil. 2011;25(1): 25-35.

18. Blood SD. The craniosacral mechanism and the temporomandibular joint. J Am Osteopath Assoc. 1986;86(8):512519 .

19. Arnadottir TS, Sigurdardottir AK. Is CranioSacral therapy effective for migraine? Tested with HIT-6 Questionnaire. Complement Ther Clin Pract. 2013;19(1):11-14.

20. Nourbakhsh MR, Fearon FJ. The effect of oscillating-energy manual therapy on lateral epicondylitis: A randomized, placebo-control, double-blinded study. J Hand Ther. 2008;21(1): 4-13.

21. Gerdner LA, Hart LK, Zimmerman MB. Craniosacral still point technique: Exploring its effects in individuals with dementia. J Gerontol Nurs. 2008;34(3):36-45.

22. Raviv G, Shefi S, Nizani D, Achiron A. Effect of CranioSacral therapy on lower urinary tract signs and symptoms in multiple sclerosis. Complement Ther Clin Pract. 2009;15(2): 72-75.

23. Castro-Sanchez AM, Lara-Palomo IC, Mataran-Penarrocha GA, Saavedra-Hernandez M, Perez-Marmol JM, AguilarFerrandiz ME. Benefits of CranioSacral therapy in patients with chronic low back pain: A randomized controlled trial. J Altern Complement Med. 2016;22(8):650-657.

24. Haller H, Lauche R, Cramer H, et al. CranioSacral therapy for the treatment of chronic neck pain: A randomized shamcontrolled trial. Clin J Pain. 2016;32(5):441-449.

25. Van der Kolk B. Body-Brain Connections. In: The Body Keeps the Score: Brain, Mind, and Body in the Healing of Trauma. New York: Penguin Books; 2014:62-64.

26. McCrory P, Meeuwisse WH, Johnston K, et al. Consensus Statement on Concussion in Sport: The 3rd International Conference on Concussion in Sport Held in Zurich, November 2008. J Athl Train. 2009;44(4):434-448.

Address correspondence to: Gail Wetzler, PT, DPT, EDO, BI-D 7853 East Arapahoe Court, Suite 1400 Centennial, CO 80116

E-mail: wetzlerdpt@gmail.com 